\title{
FERMENTAÇÃO EM ESTADO SÓLIDO EM RESÍDUOS AGROINDUSTRIAIS PARA A PRODUÇÃO DE ENZIMAS: UMA REVISÃO SISTEMÁTICA
}

\section{SOLID-STATE FERMENTATION IN AGROINDUSTRIAL RESIDUES FOR ENZYMES PRODUCTION: A SYSTEMATIC REVIEW}

P. S. dos SANTOS ${ }^{1}$, L. S. SOLIDADE ${ }^{1}$, J. G. B. SOUZA ${ }^{1}$, G. S. LIMA ${ }^{1}$, A. C. R. BRAGA Jr. ${ }^{1}$, F. G. V. de ASSIS $^{2}$ e P. L. LEAL ${ }^{1, *}$

${ }^{1}$ Universidade Federal da Bahia, Instituto Multidisciplinar em Saúde, Vitória da Conquista, Bahia, Brasil

${ }^{2}$ Centro Tecnológico de Desenvolvimento Regional de Viçosa, Ecosoluções, Viçosa, Minas Gerais, Brasil

\footnotetext{
*Patrícia Lopes Leal. Universidade Federal da Bahia, Instituto Multidisciplinar em Saúde, Vitória da Conquista, Bahia, Brasil. Phone: +55 7734292700

e-mail lealpat@yahoo.com.br(P.L.Leal).
}

\begin{tabular}{l} 
A R T I C L E I N F O \\
\hline Article history: \\
Received: 2018-02-14 \\
Accepted: 2018-03-22 \\
Available online: 2018-06-30 \\
p a l a vra - c h a ve \\
Subprodutos agroindustriais \\
bioprocessos \\
bioprodutos \\
ke yw or $d s$ \\
agroindustrial by-products \\
bioprocess \\
bioproducts
\end{tabular}

A B S T R A C T

The aim of this study was a systematic review on microbial enzyme production using solidstate fermentation (SSF) in agroindustrial residues in order to identify the main enzymes classes obtained in this process, the most applied residues as substrates and microorganisms used as fermentation agents. A total of 37 scientific articles was selected and formed a database. Approximately 49\% these articles had the brazilian nationality and indicated cellulase as the main enzyme produced by SSF in agroindustrial residues. Filamentous fungi, especially the specie Aspergillus niger, were the most applied as biological agent, wheat bran and fruit residues were the most residues used in SSF and ideal time of fermentation was the most parameter studied in process optimization. SSF is an interesting technological route to reuse of agroindustrial residues generating products with high added value.

\section{R E S U M O}

O objetivo deste trabalho foi realizar uma revisão sistemática sobre produção de enzimas microbianas sob FES em resíduos agroindustriais, a fim de identificar as principais classes de enzimas obtidas por esse processo, os resíduos mais empregados como substratos e micro-organismos utilizados como agentes da fermentação. Um total de 37 trabalhos científicos foi selecionado e formaram um banco de dados. Aproximadamente, $49 \%$ dos trabalhos foram de nacionalidade brasileira e apontaram celulase como principal enzima produzida sob FES em resíduos agroindustriais. Fungos filamentosos, especialmente a espécie Aspergillus niger, são os mais empregados como agentes biológicos, farelo de trigo e resíduos da fruticultura foram os resíduos mais estudados e tempo ideal de fermentação foi o parâmetro mais avaliado em processos de otimização. FES é uma interessante rota tecnológica para reaproveitar resíduos da agroindústria gerando produtos de alto valor agregado. 


\section{INTRODUÇÃO}

As matérias-primas lignocelulósicas são consideradas as fontes renováveis mais abundantemente encontradas na natureza, sendo compreendidas, principalmente, pelos materiais agroindustriais, pelos resíduos urbanos e pelas madeiras de angiospermas e gimnospermas (CASTRO e PEREIRA Jr., 2010). O Brasil, por apresentar uma economia fortemente ligada à agricultura, gera grandes quantidades de resíduos da agroindústria, e especial atenção vem sendo dada para o reaproveitamento desses subprodutos, contribuindo para a minimização de problemas ambientais e energéticos (COELHO et al., 2001).

Os resíduos agroindustriais, em geral, incluem os subprodutos gerados durante o processamento industrial de produtos agrícolas ou animais ou obtidos de atividades agrícolas. Normalmente, por muitas vezes não haver uma aplicação direta desses resíduos, pouco ou nenhum valor econômico é atribuído à eles, como é o caso da palha, caule, folhas, casca, semente e polpa de legumes e cereais (arroz, trigo, milho, sorgo, batata e cevada) e muitos outros. Contudo, açúcares, fibras, proteínas e minerais são compostos comumente encontrados na composição desses resíduos, o que faz deles fontes alternativas de carboidratos e nitrogênio, em substituição às fontes sintéticas desses nutrientes empregadas em bioprocessos (PANESAR et al., 2016).

Devido à composição rica em açúcares fermentáveis e nutrientes dos resíduos agroindustriais, os micro-organismos têm a capacidade de utilizar esses substratos e, subsequentemente, os converterem em vários produtos de importância industrial, como enzimas, por exemplo (MOREIRA et al., 2012; PANDA et al., 2016; RAFATULLAH et al. 2010). Neste sentido, novas rotas tecnológicas têm sido desenvolvidas para utilizar a biomassa oriunda de resíduos agroindustriais em substituição aos substratos convencionais e não renováveis empregados em bioprocessos (TAMANINI e HAULY, 2004). Vale ressaltar que além de reduzir os custos de produção e manter o rendimento proporcional ao uso de substrato sintéticos, o reaproveitamento de resíduos agroindustriais em bioprocessos contribui para a diminuição do acúmulo desses materiais no meio ambiente, que é considerado um problema ambiental atual.

Segundo RODRÍGUEZ ZÚÑIGA et al. (2011), a técnica de fermentação submersa, envolvendo na maioria das vezes micro-organismos modificados geneticamente, é responsável por quase $90 \%$ dos preparados enzimáticos industriais. No entanto, a fermentação em estado sólido (FES) vem ganhando espaço entre as pesquisas, principalmente, para produção de enzimas envolvidas na degradação de polímeros vegetais complexos, comumente presentes em resíduos agroindustriais: WISNIEWSKI et al. (2010) utilizaram farelo de soja como substrato suplementar aos resíduos do processamento de cerveja, para a produção de amilase; RODRÍGUEZ ZÚÑIGA et al. (2011) empregaram bagaço de cana de açúcar, farelo de soja, farelo de trigo e misturas entre os substratos para obtenção de celulases e endoglucanases; GUSMÃO et al. (2014) utilizaram casca de café como substrato indutor para produção de amilase, carboximetilcelulase (CMCase), avicelase e pectinase.

A fermentação em estado sólido é definida como o crescimento de micro-organismos em substratos sólidos, na ausência de água livre (RAHARDJO et al., 2006). Esta técnica tem se mostrado vantajosa, pois, além de simular o hábitat natural de fungos selvagens (HÖLKER et al., 2005, CASTRO et al., 2015; HANSEN et al., 2015), apresenta maior produtividade dos extratos enzimáticos, menor susceptibilidade à inibição e maior estabilidade das enzimas às variações de temperatura e pH (SINGHANIA et al., 2010, RODRÍGUEZ ZÚÑIGA et al., 2011). Entretanto, a FES também requer, como qualquer processo biotecnológico, controles sobre o crescimento microbiano e condições de cultivo. Algumas condições biológicas, físico-químicas e ambientais podem afetar diretamente o processo de FES e isso também vai depender do tipo de substrato e micro-organismo utilizados. Tempo de fermentação, temperatura, $\mathrm{pH}$, umidade, atividade de água, substratos e fatores nutricionais são algumas das condições envolvidas nesse processo e determinantes para alcançar uma boa produção de enzimas (KRISHNA, 2005).

Nesse contexto, o objetivo do trabalho foi realizar uma revisão sistemática sobre FES em resíduos da agroindústria a fim de investigar a eficiência desta biotecnologia na produção de enzimas, identificando as principais classes de enzimas obtidas a partir dessa técnica, micro-organismos, resíduos oriundos da agroindústria e variáveis envolvidas no processo de FES, bem como identificar aspectos que ainda devem ser melhores explorados cientificamente.

\section{MATERIAL E MÉTODOS}

\subsection{Revisão sistemática}

O levantamento bibliográfico foi conduzido no período de fevereiro a março de 2017, através de bibliotecas virtuais como PubMed, Scielo, CAPES, LILACS, dentre outras, utilizando as palavras-chave "fermentação em estado sólido", "resíduos agroindustriais" e "produção de enzimas". Os trabalhos selecionados compreenderam publicações entre os anos de 2006 a 2017 e os mesmos foram analisados de forma criteriosa para observar se a seleção correspondia aos critérios de inclusão e exclusão estabelecidos nesta revisão sistemática, conforme descrito a seguir.

\subsection{Critérios de inclusão e exclusão}

Como critérios de inclusão, optou-se: I- Os ensaios experimentais, conduzidos no Brasil ou outros países, deveriam apresentar metodologia similar; II- Os trabalhos deveriam informar sobre a produção de enzimas sob fermentação em estado sólido em resíduos da agroindústria; e III- Informações sobre a produtividade de cada tratamento nos ensaios deveriam ser relatados. O critério de exclusão aplicouse apenas aos trabalhos que não abordassem as exigências dos critérios de inclusão. Os trabalhos científicos condizentes com 
os critérios estabelecidos foram incluídos à base deste estudo e posteriormente submetidos à codificação e filtragem dos dados (LOVATTO et al., 2007; BORGES et al., 2012).

\subsection{Codificação e filtragem dos dados}

As publicações selecionadas foram analisadas criteriosamente e foram analisados quais os tipos de enzimas, resíduos e micro-organismos estudados sob FES. Após essa avaliação, filtrou-se apenas os estudos referentes a esses requisitos e analisou-se criticamente as variáveis envolvidas no processo de fermentação, como: tempo de fermentação, temperatura, $\mathrm{pH}$, teor de água, atividade de água e elementos adicionais (suplementação e tratamento dos meios). Esses dados foram tabulados e foi avaliada a influência deles em relação à produção enzimática (LOVATTO et al., 2007).

\section{RESULTADOS E DISCUSSÃO}

A partir do levantamento bibliográfico, empregando os critérios de inclusão e exclusão, foram selecionadas 39 publicações sobre produção de enzimas microbianas em FES utilizando resíduos da agroindústria como substratos (Tabela $1)$.

Tabela 1. Banco de publicações científicas selecionadas de acordo com os critérios de inclusão e exclusão estabelecidos.

\begin{tabular}{|c|c|c|}
\hline \multicolumn{3}{|c|}{ Publicações científicas selecionadas } \\
\hline $\begin{array}{c}\text { Código do } \\
\text { trabalho }\end{array}$ & Autores/Ano & País \\
\hline 1 & NOVELLI et al. (2016) & Brasil \\
\hline 2 & AHMED e MOSTAFA (2013) & Egito \\
\hline 3 & SWAIN et al. (2009) & Índia \\
\hline 4 & TEPE e DURSUN (2014) & Alemanha \\
\hline 5 & MURTHY et al. (2009) & Índia \\
\hline 6 & SANTOS et al. (2012) & Brasil \\
\hline 7 & TAO et al. (2011) & China \\
\hline 8 & SALIHU et al. (2015) & Nigéria \\
\hline 9 & CRUZ et al. (2011) & Brasil \\
\hline 10 & RAZA et al. (2011) & Paquistão \\
\hline 11 & RODRÍGUEZ-ZÚÑIIGA et al. (2011) & Brasil \\
\hline 12 & SANTOS et al. (2013a) & Brasil \\
\hline 13 & MRUDULA E MURUGAMMAL (2011) & Índia \\
\hline 14 & REDDY et al. (2015) & Índia \\
\hline 15 & SOUZA e AQUINO (2012) & Brasil \\
\hline 16 & PENHA et al. (2016) & Brasil \\
\hline 17 & DANTAS e AQUINO (2010) & Brasil \\
\hline 18 & $\begin{array}{l}\text { MAHALAKSHMI e JAYALAKSHMI } \\
(2016)\end{array}$ & Índia \\
\hline 19 & MRUDULA e ANITHARAJ (2011) & Índia \\
\hline 20 & KANIMOZHI e NAGALAKSHMI (2014) & Índia \\
\hline 21 & BANSAL et al. (2012) & Índia \\
\hline 22 & NCUBE et al. (2012) & África do Sul \\
\hline 23 & SETHI et al. (2015) & Índia \\
\hline 24 & MADHUMITHAH et al. (2011) & Índia \\
\hline 25 & SANTOS et al. (2014) & Brasil \\
\hline 26 & SOUZA et al. (2010) & Brasil \\
\hline 27 & SUGANTHI et al. (2011) & Índia \\
\hline 28 & SANTOS et al. (2013b) & Brasil \\
\hline 29 & SANTOS et al. (2011) & Brasil \\
\hline 30 & SOUZA et al. (2015) & Brasil \\
\hline 31 & PARIS et al. (2012) & Brasil \\
\hline
\end{tabular}

\begin{tabular}{|l|l|l|}
\hline 32 & CASTRO e PINTO (2009) & Brasil \\
\hline 33 & NOVAKI et al. $(2010)$ & Brasil \\
\hline 34 & DELABONA et al. $(2009)$ & Brasil \\
\hline 35 & LEE et al. $(2014)$ & Coréia do Sul \\
\hline 36 & PACHECO et al. $(2012)$ & Brasil \\
\hline 37 & SAHA et al. $(2014)$ & Índia \\
\hline 38 & ERGUN e UREK (2017) & Turquia \\
\hline 39 & COSTA et al. $(2017)$ & Brasil \\
\hline
\end{tabular}

Do total de publicações selecionadas, o Brasil deteve, aproximadamente, $49 \%$ dos estudos sobre produção de enzimas microbianas utilizando resíduos da agroindústria sob FES. Por ser um país essencialmente agrícola, o Brasil se caracteriza como um dos maiores produtores mundiais de biomassa e consequentemente, um grande volume de resíduos orgânicos é gerado pela agroindústria (SOUSA et al., 2011). Segundo informação do Plano Nacional de Resíduos Sólidos (2011), um total de 290.838.411 toneladas/ano é produzido anualmente no Brasil.

Esse cenário tem estimulado os setores públicos e privados a buscarem tecnologias para o aproveitamento e valorização de resíduos agroindustriais, tal como o processo de fermentação em estado sólido para a produção de enzimas. Considerando o período entre os anos de 2006 a 2017, nenhuma publicação científica, anterior ao ano de 2009, abordando produção de enzimas microbianas utilizando resíduos da agroindústria sob FES, foi encontrada nas bibliotecas virtuais determinadas nesta revisão (Figura 1). A partir do ano de 2009, observou-se números consideráveis de publicações sobre o tema, especialmente no ano de 2011 (9 publicações). Isso pode ser explicado pelo aumento da preocupação em reduzir os resíduos agroindustriais do meio ambiente, conforme mencionado por PACHECO et al. (2012) que destaca a importância do reaproveitamento desses resíduos em processos industriais, visando inclusive a sustentabilidade ambiental.

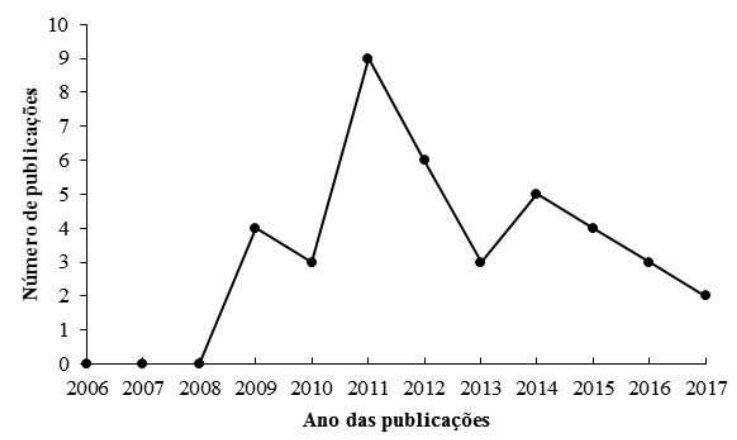

Figura 1. Número de publicações sobre produção de enzimas microbianas utilizando resíduos da agroindústria sob FES compreendido entre os anos de 2006 a 2017, de acordo com o banco de dados de publicações.

Diferentes resíduos agroindustriais foram empregados em FES nos estudos selecionados, sendo o farelo de trigo amplamente utilizado nas pesquisas. Das 37 publicações selecionadas, $46 \%$ estudaram farelo de trigo, conforme ilustrado na Figura 2. 


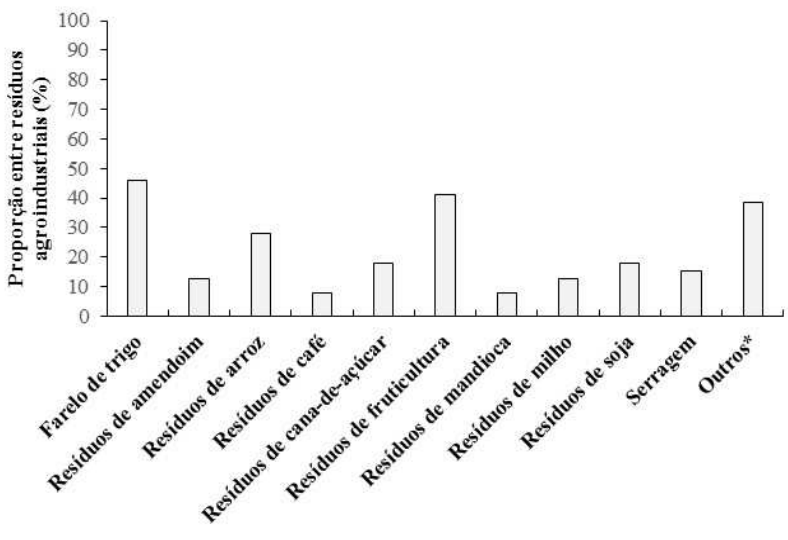

Figura 2. Proporção entre resíduos da agroindústria utilizados como substratos em FES para produção de enzimas de acordo com o banco de dados.

*Outros = polpa de beterraba, batata, casca de batata, casca de sorgo, resíduos de chá, resíduos do óleo de dendê, sementes de abóbora, farelo de grama preta, casca de palha, casca de cenoura, casca de grama verde, resíduos de berinjela, bolo de óleo de coco, folhas vegetais, torta de girassol.

Resíduos da fruticultura também se destacaram como substratos alternativos em FES (41\%), em especial cascas, polpas, bagaço e sementes de frutas. BANSAL et al. (2012) observaram que farelo de trigo foi o substrato que melhor proporcionou a produção de celulases $\left(310,6 ; 16,8\right.$ e 33,0 U.g ${ }^{-1}$ de matéria seca do substrato para CMCase, FPase e $\beta$ glucosidase, respectivamente) quando comparado aos resíduos de milho, frutas, arroz, cana-de-açúcar, dentre outros. Por outro lado, MRUDULA e MURUGAMMAL (2011) verificaram que resíduo de coco foi o substrato que levou a máxima produção de celulases $\left(3,42\right.$ e 1,77 U.g ${ }^{-1}$ para CMCase e FPase, respectivamente) em relação a resíduos de arroz, farelo de trigo e serragem.

Os resíduos agroindustriais, de uma forma geral, apresentam em sua composição celulose, hemicelulose e lignina (TAMANINI e HAULY, 2004), o que justifica o fato de celulase e pectinase ocuparem posição de destaque dentre as enzimas mais produzidas em FES utilizando esses resíduos como substrato (Figura 3).

Do total de trabalhos avaliados, celulase foi alvo de investigação de $41 \%$ dos estudos, seguida de pectinase (16\%). Além da composição lignocelulósica dos resíduos agroindustriais contribuir para obtenção dessas enzimas a partir de FES, há um amplo interesse pela produção de celulase e pectinase devido às suas aplicações com potencial biotecnológico para diferentes setores da indústria: combustível, produtos têxteis, papel, química, ração animal, dentre outros (NCUBE et al., 2012).

De acordo com as publicações selecionadas neste trabalho, fungos filamentosos foram produtores de protease, pectinase, celulase, lipase, amilase, tanase, invertase e xilanase, utilizando resíduos agroindustriais como substrato em FES, enquanto que bactérias produziram, especialmente, pectinase, galactosidase e amilase.

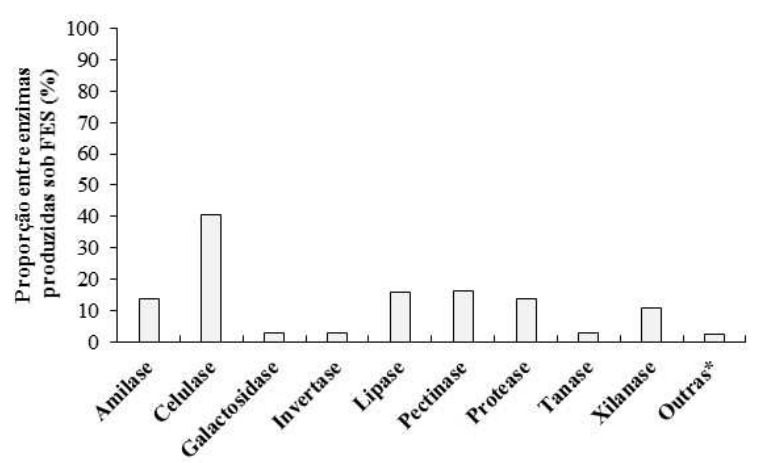

Figura 3. Proporção entre as enzimas produzidas a partir de FES em resíduos da agroindústria de acordo com o banco de dados.

*Outras = Manganês peroxidase, lignina peroxidase e aril-álcool oxidase.

Diversas espécies microbianas têm sido cultivadas sob FES em resíduos agroindustriais objetivando a produção de enzimas. No entanto, fungos filamentosos têm merecido uma atenção especial, comparado às bactérias, uma vez que devido às propriedades fisiológicas, bioquímicas e enzimáticas, os fungos conseguem se adaptar à baixa umidade, crescendo facilmente sobre o substrato (ZEN et al., 2014). Isto explica a preferência por fungos filamentosos como agentes produtores de enzimas sob FES em resíduos agroindustriais nas investigações dos estudos selecionados, sendo o gênero Aspergillus o mais explorado (Figura 4).

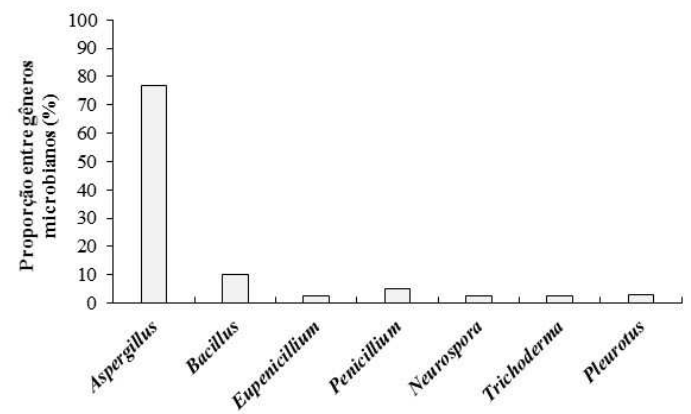

Figura 4. Proporção entre gêneros de micro-organismos produtores de enzimas sob FES em resíduos agroindustriais investigados, acordo com o banco de dados.

Vale ressaltar ainda que, Aspergillus niger (A. niger) foi a espécie mais abordada pelos estudos, totalizando $77 \%$ das avaliações (Figura 5). Essa espécie foi amplamente utilizada principalmente na produção de celulases, uma vez que faz parte do gênero que é considerado como degradante primário de celulose e lignina (AGUIAR, 2010). SALIHU et al. (2015) e SANTOS et al. (2013b) empregaram A. niger para a produção de celulases e obtiveram valores máximos de produção registrados sob FES quando utilizaram palha de arroz como substrato $\left(1,76 ; 1,22\right.$ e $0,91{\mathrm{U} . g^{-1}}^{-1}$ para CMCase, FPase e $\beta$-glucosidase, respectivamente) e farelo de cacau $(14,18 ; 7,51$ U.mL ${ }^{-1}$ e FPase, respectivamente). 


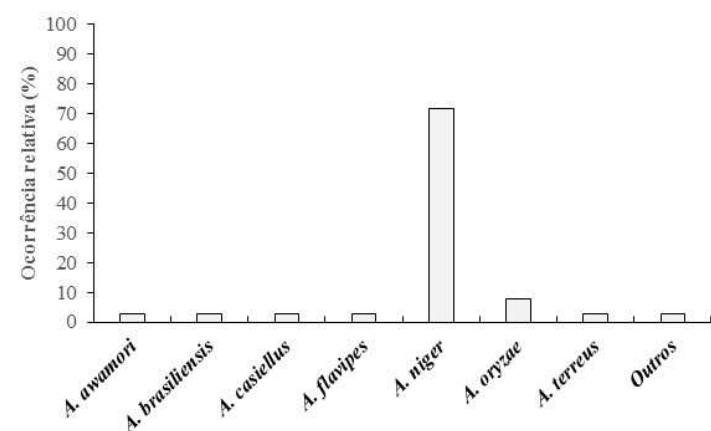

Figura 5. Proporção entre espécies de Aspergillus empregadas no banco de dados para produção de enzimas sob fermentação em estado sólido em resíduos agroindústriais.

RAZA et al. (2011) analisaram três espécies de Aspergillus (A. niger, A. oryzae e A. awamori) para a produção de $\beta$-glucosidase (celulase) e verificaram para a espécie Aspergillus oryzae a máxima produção da enzima (875 U.g $\left.{ }^{-1}\right)$. REDDY et al. (2015), SANTOS et al. (2012) e MRUDULA e MURUGAMMAL (2011) utilizaram A. niger do tipo selvagem para a produção de celulase. COSTA et al. (2017) verificaram atividade máxima de lipase igual $19.8 \mathrm{U} \cdot \mathrm{g}^{-1}$ para $A$. niger selvagem cultivado sob FES em resíduos agroindustriais, enquanto SANTOS et al. (2014) reportaram máxima produção

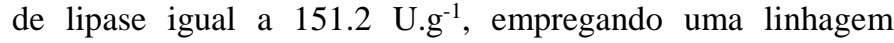
mutante de $A$. niger sob cultivo em FES. De fato, alguns estudos têm sugerido melhores rendimentos de produção de enzimas a partir de fungos filamentosos mutantes sob cultivo em estado sólido utilizando resíduos agroindustriais como substrato (SOUZA et al., 2010; SANTOS et al., 2013b).

A eficiência da FES para produção de enzimas vai além das escolhas corretas de substrato e agente biológico, sendo necessárias as adequações dos parâmetros físico-químicos do processo. De acordo com PIROTA et al. (2015), a FES tem demonstrado eficiência no crescimento de fungos filamentosos e gera maiores rendimentos enzimáticos quando comparada a fermentação submersa (FS), ocorridas em meio líquido. Entretanto, ainda existem limitações relacionadas a esse processo fermentativo que podem afetar a produção enzimática, como a reprodutibilidade dos resultados e regulação e efeitos negativos sobre variáveis envolvidas e relacionados às condições estáticas (HÖLKER e LENZ, 2005).

Para os estudos selecionados neste trabalho, foram verificados seis parâmetros ambientais submetidos à otimização do processo fermentativo: tempo de fermentação; temperatura; pH; teor de água; atividade de água e enriquecimento nutricional (Figura 6). Destes parâmetros, o tempo ideal de fermentação foi avaliado em $97 \%$ dos trabalhos selecionados e atividade de água foi o parâmetro menos otimizado. SWAIN et al. (2009) observaram que o melhor tempo de fermentação para a produção de pectinase por Bacillus subitilis CM5 foi de 144 horas. Por outro lado, MRUDULA e ANITHARAJ (2011) verificaram que 96 horas foi o melhor tempo de fermentação para a produção da mesma enzima a partir de $A$. niger.

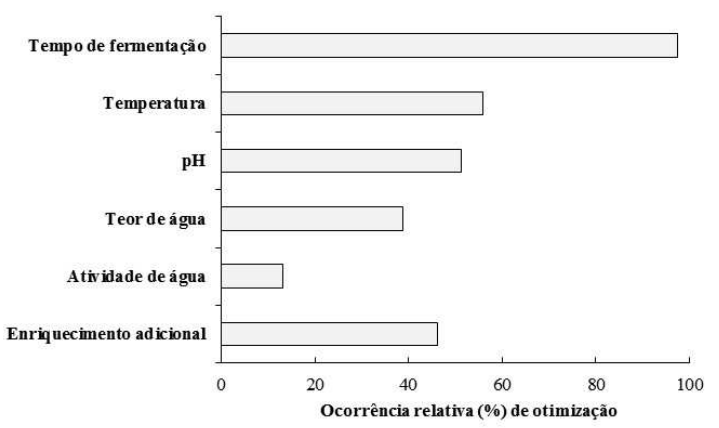

Figura 6. Proporção entre as variáveis ambientais otimizadas em processos de FES para produção de enzimas, de acordo com o banco de dados.

O tempo de fermentação está diretamente relacionado com o esgotamento nutricional no meio, implicando na redução do crescimento microbiano, inviabilizando a conversão da biomassa em produtos e, consequentemente, reduzindo a produção de enzimas (SANTOS et al., 2013b). O $\mathrm{pH}$ pode influenciar no transporte de componentes na membrana das células (KAPOOR et al., 2008) e assim, induzir mudanças morfológicas nos micro-organismos e interferir na estabilidade enzimática (MRUDULA e MURUGAMMAL, 2011). A temperatura está relacionada com o transporte de massa e com a transferência de oxigênio (WANG e YANG, 2007). Em FES, a umidade está relacionada ao teor e atividade de água, sendo que o teor de água deve ser ajustado para que não ocasione contaminações no meio de fermentação devido a uma alta umidade ou proporcione o surgimento de metabólitos devido a uma baixa umidade, enquanto que a atividade de água deve ser determinada de acordo com a necessidade de água dos micro-organismos utilizados, no qual a atividade livre permite reações sobre o substrato sólido (AFONSO, 2012). O enriquecimento e o pré-tratamento dos substratos têm sido adotados em FES como alternativas para contribuir com um maior fornecimento de nutrientes, permitindo que os microorganismos cresçam mais facilmente: COSTA et al. (2017) optaram pelo enriquecimento de resíduos agroindustriais (farelos de arroz, trigo e soja) com glicose, glicerol, óleo de soja ou óleo de trigo e obtiveram produção máxima de lipase por A. niger cultivado em mistura de farelo de arroz e glicerol (19.844 U.g-1). ERGUN e UREK (2017) conduziram vários pré-tratamentos envolvendo $\mathrm{NaOH}$ ou água destilada para utilização de batatas como substrato para produção de enzimas por $P$. ostreatus e verificaram maior atividade lignocelulolítica $\left(6708.3 \pm 75\right.$ U.L $\left.{ }^{-1}\right)$ empregando água destilada no prétratamento.

BANSAL et al. (2012) observaram que para produção máxima de CMCase, FPase e $\beta$-glucosidase, a partir de FES em farelo de trigo, foi obtida sob $30^{\circ} \mathrm{C}, \mathrm{pH}$ igual 7.0 e tempo de fermentação de 96 horas e relação substrato/água igual 1:1,5 e 1:1,75. Enquanto que SANTOS et al. (2011) verificaram que os melhores valores de atividade de água para a produção de celulases, usando Aspergillus niger, em resíduos de manga como substrato, foram: 0,929 Aw para CMCase $\left(7,26{\left.\mathrm{U} . \mathrm{g}^{-1}\right) \mathrm{e}}^{\mathrm{N}}\right.$ 


\section{0,927 Aw para FPase (2,55 U.g $\left.{ }^{-1}\right)$.}

SOUZA et al. (2015) além de terem estudado variáveis como temperatura, tempo de incubação, $\mathrm{pH}$ e teor de água, observaram o efeito do enriquecimento do meio fermentativo com alguns componentes, no qual a suplementação com nitrato de potássio $2.71 \%\left(\mathrm{w} . \mathrm{w}^{-1}\right)$ e ácido tânico $1.53 \%\left(\mathrm{w} \cdot \mathrm{w}^{-1}\right)$ permitiram efeitos significativos na produção de tanase por Aspergillus sp. GM4. TAO et al. (2011) avaliaram o efeito de suplementação nutricional com várias fontes de nitrogênio $\left(\mathrm{NH}_{4}\right)_{2} \mathrm{SO}_{4}, \mathrm{KNO}_{3},\left(\mathrm{NH}_{2}\right)_{2} \mathrm{CO}$, dentre outros - e sais minerais inorgânicos - $\mathrm{CaCl}_{2}, \mathrm{MgSO}_{4}, \mathrm{Na}_{3} \mathrm{PO} 4$, dentre outros - para a produção de xilanase, pectinase e celulase a partir de Eupenicillium javanicum utilizando resíduo de laranja. SALIHU et al. (2015) estudaram diversos resíduos com e sem tratamento, no qual os melhores valores de atividade de celulases foram encontrados para o resíduo casca se soja que foi submetido a tratamento alcalino $\left(9,91 ; 6,20\right.$ e 5,69 ${\mathrm{U} . \mathrm{g}^{-1}}^{-1}$ para CMCase, FPase e $\beta$-glucosidase, respectivamente).

\section{CONCLUSÕES}

O Brasil foi o principal país gerador de publicações, na última década, sobre produção de enzimas a partir de FES em resíduos agroindustriais. Farelo de trigo e resíduos advindos da fruticultura foram os mais empregados como substratos na FES para a obtenção de enzimas. De forma geral, os trabalhos selecionados nesta revisão optaram pela celulase como enzima a ser produzida por FES em resíduos da agroindústria e Aspergillus niger como o agente biológico utilizado nesse processo. A otimização do processo em FES tem sido amplamente conduzido a fim de alcançar um melhor rendimento na produção de enzimas. A utilização de resíduos agroindustriais como substratos em FES para produção de enzimas representa ainda uma rota tecnológica em experimentação, porém com potencial aplicação em grandes escalas, o que contribuirá, substancialmente, com a minimização dos custos de produção, com a redução do acúmulo desses materiais no meio ambiente e agregação de valores à matérias primas, até pouco tempo, desvalorizadas.

\section{R E F E R E N C I A S}

AFONSO, L.C. Produção de celulases por cultivo em estado sólido e aplicação na hidrólise de bagaço de cana-deaçúcar. 2012. 120f. Dissertação (Mestrado em Engenharia) - Escola Politécnica, Universidade de São Paulo, São Paulo, 2012.

AGUIAR, C.M. Hidrólise enzimática de resíduos lignocelulósicos utilizando celulases produzidas pelo fungo Aspergillus niger. 2010. 118f Dissertação (Mestrado em Engenharia Química) - Centro de Engenharias e Ciências Exatas, Universidade Estadual do Oeste do Paraná, Toledo, 2010.

AHMED, S.A.; MOSTAFA, F.A. Utilization of orange bagasse and molokhia stalk for production of pectinase enzyme. Brazilian Journal of Chemical Engineering, v.30, n.3, p.449-456, 2013.
Brasil, Ministério do Meio Ambiente. Plano Nacional de Resíduos Sólidos. Versão Preliminar para Consulta Pública, setembro de 2011. Disponível em: http://www.mma.gov.br/estruturas/253/_publicacao/253_p ublicacao02022012041757.pdf. Acesso em: 09 Mai. 2016.

BANSAL, N.; TEWARI, R.; SONI, R.; SONI, S.K. Production of cellulases from Aspergillus niger NS-2 in solid state fermentation on agricultural and kitchen waste residues. Waste management, v.32, p.1341-1346, 2012.

BORGES, G.F.; TEIXEIRA, A.M.; FERREIRA, J.P. Meta-análise do efeito no sistema imunitário da suplementação de hidratos de carbono no exercício físico. Motricidade, v.8, n.2, p.83-97, 2012.

CASTRO, A.M.; CASTILHO, L.R.; FREIRE, D.M.G. Performance of a fixed-bed solid-state fermentation bioreactor with forced aeration for the production of hydrolases by Aspergillus awamori. Biochemical Engineering Journal, v.93, p.303-308, 2015.

CASTRO, A.N.; PEREIRA Jr., N. Produção, propriedades e aplicação de celulases na hidrólise de resíduos agroindustriais. Química Nova, v. 33, n.1, p.181-188, 2010.

CASTRO, R.J.S.; PINTO, G.A.S. Estudo comparativo da produção de proteases por Aspergillus oryzae em fermentação semi-sólida utilizando tortas de girassol. In: SIMPÓSIO NACIONAL DE BIOPROCESSOS, 17, 2009, Natal. Anais... Natal: Universidade Federal do Rio Grande do Norte, 2009.

COELHO, M.A.Z., LEITE, S.G.F.; ROSA, M.F.; FURTADO, A.A.L. Aproveitamento de Resíduos Agroindustriais: Produção de Enzimas a Partir da Casca de Coco Verde. B.CEPPA, v.19, n.1, p. 33-42, 2001.

COSTA, T.M., HERMANN, K.L., GARCIA-ROMAN, M., VALLE, R. DE C.S.C., TAVARES, L.B.B. lipase production by aspergillus niger grown in different agroindustrial wastes by solid-state fermentation. Brazilian Journal of Chemical Engineering, v. 34, n. 2, p. 419 427, 2017.

CRUZ, E.A.; MELO, M.C.; SANTANA, N.B.; FRANCO, M.; SANTANA, R.S.M.; SANTOS, L.S.; GONÇALVES, Z.S. Produção de alfa-Amilase por Aspergillus niger em resíduo de cascas de mandioca. Journal of Health Sciences, v.13, n.4, p.245-9, 2011.

DANTAS, E.M.; AQUINO, L.C.L. Fermentação em estado sólido de diferentes resíduos para a obtenção de lipase microbiana. Revista Brasileira de Produtos Agroindustriais. v.12, n.1, p.81-87, 2010.

DELABONA, P. das S.; PIROTA, R.D.P.B.; PINTO, G.A.S.; FARINAS, C.S. Produção de celulases e xilanases por duas linhagens de Trichoderma visando a aplicação na produção de etanol celulósico. In: Congresso Internacional de Bioenergia, 4, 2009, Curitiba, Anais... Curitiba: Fupef, 2009.

ERGUN, S.O., UREK, R.O. Production of ligninolytic enzymes by solid state fermentation using Pleurotus ostreatus. Annals of Agrarian Science, v. 15, p. 273-277, 2017.

GUSMÃO, R.O.; FERRAZ, L. M.; RÊGO, A. P. B; ASSIS, F. G. V.; LEAL, P.L. Produção de enzimas por Aspergillus spp. sob fermentação em estado sólido em casca de café. Scientia Plena, v.10, n.11, p. 116-202, 2014.

HANSEN, G.H.; LÜBECK, M.; FRISVAD, J.C.; LÜBECK, P.S.; ANDERSEN, B. Production of cellulolytic enzymes from ascomycetes: comparison of solid state and submerged 
fermentation. Process Biochemistry, v.50, n.9, p.13271341, 2015.

HÖLKER, U.; LENZ, J. Solid-state fermentation - are there any biotechnological advantages? Current Opinion in Microbiology, v.8, n.3, p.301-306, 2005.

KANIMOZHI, K.; NAGALAKSHMI, P.K. Xylanase production from Aspergillus niger by solid state fermentation using agricultural waste as substrate. Int. J. Curr. Microbiol. App. Sci., v.3, n.3, p.437-446, 2014.

KAPOOR, M.; NAIR, L.M.; KUHAD, R.C. Cost-effective xylanase production from free and immobilized Bacillus pumilus strain MK001 and its application in saccharification of Prosopis juliflora. Biochemical Engineering Journal, v.38, n.1, p.88-97, 2008.

KRISHNA, C. Solid-state fermentation systems - an overview. Critical Reviews in Biotechnology, v.25, n.1-2, p.1-30, 2005.

LEE, J.; PARK, I.; CHO, J. Production of extracellular $\alpha$ galactosidase by Bacillus sp. LX-1 in solid state fermentation for application as a potential feed additive. Revista Colombiana de Ciencias Pecuarias, v.27, n.3, p.194-201, 2014.

LOVATTO, P.A.; LEHNEN, C.R.; ANDRETTA, I.; CARVALHO, A.D.; HAUSCHILD, L. Meta-análise em pesquisas científicas-enfoque em metodologias. Revista Brasileira de Zootecnia, v.36, p.285-294, 2007.

MADHUMITHAH, C.G.; KRITHIGA, R.; SUNDARAM, S.; SASIKUMAR, C.S.; GUHATHAKURTA, S.; CHERIAN, K.M. Utilization of vegetable wastes for production of protease by solid state fermentation using Aspergillus niger. World Journal of Agricultural Sciences, v.7, n.5, p.550-555, 2011.

MAHALAKSHMI, N.; JAYALAKSHMI, S. Cellulase production by Aspergillus niger under solid state fermentation using agro industrial wastes. Int. J. Adv. Multidiscip. Res., v.3, n. 2, p. $78-83,2016$.

MOREIRA, L.R.; DE S.; FERREIRA, G.V.; SANTOS, S.S.T.; RIBEIRO, A.P.S.; SIQUEIRA, F.G.; FILHO, E.X.F. The hydrolysis of agro-industrial residues by holocellulosedegrading enzymes. Brazilian Journal of Microbiology, v.43, n.2, p.498-505, 2012.

MRUDULA, S.; ANITHARAJ, R. Pectinase production in solid state fermentation by Aspergillus niger using orange peel as substrate. Glob. J. Biotechnol. Biochem., v.6, n.2, p.64-71, 2011

MRUDULA, S.; MURUGAMMAL, R. Production of cellulase by Aspergillus niger under submerged and solid state fermentation using coir waste as a substrate. Brazilian Journal of Microbiology, v.42, n.3, p.1119-1127, 2011.

MURTHY, P.S.; NAIDU, M.M.; SRINIVAS, P. Production of $\alpha$-amylase under solid-state fermentation utilizing coffee waste. Journal of Chemical Technology and Biotechnology, v.84, n.8, p.1246-1249, 2009.

NCUBE, T.; HOWARD, R.L.; ABOTSI, E.K.; RENSBURG, E.L.J.; NCUBE, I. Jatropha curcas seed cake as substrate for production of xylanase and cellulase by Aspergillus niger FGSCA733 in solid-state fermentation. Industrial Crops and Products, v.37, n.1, p.118-123, 2012.

NOVAKI, L.; HASAN, S.D.M.; KADOWAKI, M.K.; ANDRADE, D. Produção de invertase por fermentação em estado sólido a partir de farelo de soja. Engevista, v.12, n.2, p.131-140, 2010.
NOVELLI, P.K.; BARROS, M.M.; FLEURI, L.F. Novel inexpensive fungi proteases: production by solid state fermentation and characterization. Food Chemistry, v.198, p.119-124, 2016.

PACHECO, C.S.V.; FERREIRA, A.N.; ROCHA, T.J.O.; TAVARES, I.M. DE C.; FRANCO, M. Aproveitamento da semente da jaca para a obtenção de endoglucanase a partir de Aspergillus niger por fermentação em estado sólido. Journal of Health Sciences, v.14, n.1, p.25-9, 2012.

PANDA, S.K.; MISHRA, S.S.; KAYITESI, E.; RAY, R.C. Microbial-processing of fruit and vegetable wastes for production of vital enzymes and organic acids: Biotechnology and scopes. Environmental research, v. 146, p.161-172, 2016.

PANESAR, P.S., KAUR, R., SINGLA, G., SANGWAN, R.S. Bio-processing of agro-industrial wastes for production of food-grade enzymes: progress and prospects. Appl Food Biotechnol, v. 3, n. 4, p. 208-227, 2016.

PARIS, L.D.; SCHEUFELE, F.B.; JÚNIOR, A.T.; GUERREIRO, T.L.; HASAN, S.D.M. Produção de complexos enzimáticos por A. niger a partir de soja por fermentação em estado sólido. Acta Scientiarum Technology, v.34, n.2, p.193-200, 2012.

PENHA, E. DAS M.; VIANA, L. DE A.N.; GOTTSCHALK, L.M.F.; TERZI, S. DA C.; SOUZA, E.F.; FREITAS, S.C.; SANTOS, J.DE O.; SALUM, T.F.C. Aproveitamento de resíduos da agroindústria do óleo de dendê para a produção de lipase por Aspergillus niger. Ciência Rural, v.46, n.4, p.755-761, 2016.

PIROTA, R.D.P.B.; TONELOTTO, M.; DELABONA, P. DA S.; TREMACOLDI, C.R.; FARINAS, C.S. Caracterização de fungos isolados da região Amazônica quanto ao potencial para produção das enzimas envolvidas na conversão da biomassa vegetal. Ciência Rural, v.45, n.9, p.1606-1612, 2015.

RAFATULLAH, M.; SULAIMAN, O.; HASHIM, R.; AHMAD, A. Adsorption of methylene blue on low-cost adsorbents: a review. Journal of hazardous materials, v.177, n.1, p.70$80,2010$.

RAHARDJO, Y.S.P.; TRAMPER, J.; RINZEMA, A. Modeling conversion and transport phenomena in solid-state fermentation: a review and perspectives. Biotechnology Advances, v.24, p.161-179, 2006.

RAZA, F.; RAZA, N.A.; HAMEED, U.; HAQ, I.; MARYAM, I. Solid state fermentation for the production of $\beta$ glucosidase by co-culture of Aspergillus niger and $A$. oryzae. Pak. J. Bot., v.43, n.1, p.75-83, 2011.

REDDY, G.P.K.; NARASIMHA, G.; KUMAR, K.D.; RAMANJANEYULU, G.; RAMYA, A.; KUMARI, B.S.S.; REDDY, B.R. Cellulase production by Aspergillus niger on different natural lignocellulosic substrates. International Journal of Current Microbiology and Applied Sciences, v.4, n.4, p.835-845, 2015.

RODRÍGUEZ-ZÚÑIGA, U.F.; FARINAS, C.S.; NETO, V.B.; COURI, S.; CRESTANA, S. Produção de celulases por Aspergillus niger por fermentação em estado sólido. Pesquisa Agropecuária Brasileira, v.46, n.8, p.912-919, 2011.

SAHA, K.; MAITY, S.; ROY, S.; PAHAN, K.; PATHAK, R.; MAJUMDAR, S.; GUPTA, S. Optimization of amylase production from B. amyloliquefaciens (MTCC 1270) using solid state fermentation. International journal of microbiology, v.2014, p.1-7, 2014. 
SALIHU, A.; ABBAS, O.; SALLAU, A.B.; ALAM, M.Z. Agricultural residues for cellulolytic enzyme production by Aspergillus niger: effects of pretreatment. Biotech., v.5, n.6, p.1101-1106, 2015.

SANTOS, R.R.; MURUCI, L.N.M.; DAMASO, M.C.T.; SILVA, J.P.L.; SANTOS, L.O. Lipase production by Aspergillus niger 11T53A14 in wheat bran using experimental design methodology. Journal of Food and Nutrition Research., v.2, n.10, p.659-663, 2014.

SANTOS, T.C.; CAVALCANTI, I.S.; BONOMO, R.C.F.; SANTANA, N.B.; FRANCO, M. Optimization of productions of cellulolytic enzymes by Aspergillus niger using residue of mango a substrate. Ciência Rural, v.41, n.12, p.2210-2216, 2011.

SANTOS, T.C.; FILHO, G.A.; ROCHA, T.J.H.; FERREIRA, A.N.; DINIZ, G.A.; FRANCO, M. Produção e quantificação de celulases por meio da fermentação em estado sólido de resíduos agroindustriais. Scientia Agraria Paranaensis, v.12, n.2, p.115-123, 2013a.

SANTOS, T.C.; GOMES, D.P.P.; BONOMO, R.C.F.; FRANCO, M. Optimisation of solid state fermentation of potato peel for the production of cellulolytic enzymes. Food Chemistry, v.133, n.4, p.1299-1304, 2012.

SANTOS, T.C.; ROCHA, T.J.O.; OLIVEIRA, A.C.; FILHO, G.A.; FRANCO, M. Aspergillus niger como produtor de enzimas celuloliticas a partir farelo de cacau (Theobroma cacao). Arq. Inst. Biol., v.80, n.1, p.65-71, 2013 b.

SETHI, B.K.; JANA, A.; NANDA, P.K.; MOHAPATRA, P.K.; SAHOO, S.L. Thermostable acidic protease production by Aspergillus terreus NCFT 4269.10 using chickling vetch peels. Journal of Taibah University for Science, v.10, n.4, p.571-583, 2015.

SINGHANIA, R.R.; SUKUMARAN, R.K.; PATEL, A.K.; LARROCHE, C.; PANDEY, A. Advancement and comparative profiles in the production technologies using solid-state and submerged fermentation for microbial cellulases. Enzyme and Microbial Technology, v.46, p.541-549, 2010.

SOUSA, M.S.B.; VIEIRA, L.M.; SILVA, M. DE J.M.; LIMA, A Caracterização nutricional e compostos antioxidantes em resíduos de polpas de frutas tropicais. Ciênc. Agrotec., v.35, n.3, p.554-559, 2011.

SOUZA, F.M.; AQUINO, L.C.L. Potencial da farinha de sementes de mangaba para a produção de lipase de Aspergillus niger: Influência da temperatura e umidade no processo. Scientia Plena, v.8, n.12, p.1-5, 2012.

SOUZA, P.N. DA C.; MAIA, N. DA C.; GUIMARÃES, L.H.S.; RESENDE, M.L.V.; CARDOSO, P.G. Optimization of culture conditions for tannase production by Aspergillus sp. GM4 in solid state fermentation. Acta Scientiarum Biological Sciences, v.37, n.1, p.23-30, 2015.

SOUZA, R.L.A.; OLIVEIRA, L.S.C.; SILVA, F.L.H.; AMORIM, B.C. Caracterização da poligalacturonase produzida por fermentação semi-sólida utilizando-se resíduo do maracujá como substrato. Revista Brasileira de Engenharia Agricola e Ambiental, v.14, n.9, p.987-992, 2010.

SUGANTHI, R.; BENAZIR, J.F.; SANTHI, R.; KUMAR, V.R.; HARI, A.; MEENAKSHI, N.; NIDHIYA, K.A.; KAVITHA, G.; LAKSHMI, R. Amylase production by Aspergillus niger under solid state fermentation using agro industrial wastes. International Journal of Engineering Science and Technology, v.3, n.2, p.1756-1763, 2011.

SWAIN, M.R.; KAR, S.; RAY, R.C. Exo-polygalacturonase production by Bacillus subtilis CM5 in solid state fermentation using cassava bagasse. Brazilian Journal of Microbiology, v.40, n.3, p.636-648, 2009.

TAMANINI, C.; HAULY, M.C. DE O. Resíduos agroindustriais para produção biotecnológica de xilitol. Semina: Ciências Agrárias, v.25, n.4, p.315-330, 2004.

TAO, N.; SHI, W.; LIU, Y.; HUANG, S. Production of feed enzymes from citrus processing waste by solid-state fermentation with Eupenicillium javanicum. International Journal of Food Science \& Technology, v.46, n.5, p.1073-1079, 2011.

TEPE, O.; DURSUN, A.Y. Exo-pectinase production by Bacillus pumilus using different agricultural wastes and optimizing of medium components using response surface methodology. Environmental Science and Pollution Research, v.21, n.16, p.9911-9920, 2014.

WANG, W.; YANG, S. Solid State Fermentation and Its Applications. In: Yang, S. (ed.). Bioprocessing for valueadded products from renewable resources. Elsevier, 2007. cap.18, p.465-489.

WISNIEWSKI, A.C, DE ALMEIDA, M.A.L, PALMA, M.P., TAVARES, L.B.B. Produção de enzimas amilolíticas por Macrocybe titans em resíduo do processamento de cerveja. Revista Brasileira de Biociências, v.8, p.285-293, 2010.

ZEN, C.K.; SILVA, K.P.; REINEHR, C.O.; BERTOLIN, T.E.; COLLA, L. M. Introdução da síntese de lipídios e proteínas por Aspergillus niger. Revista CIATEC-UPF, v.6, n.2, p.40-47, 2014. 